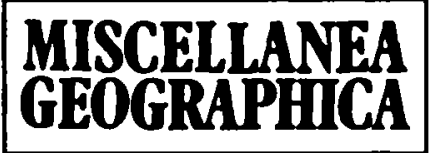

WARSZAWA 1992 Vol. 5

Jerzy Gilarowski

\title{
ESSAI DE RECONSTITUTION DE L'ÉTENDUE DE LA FORÊT DENSE HUMIDE EN AFRIQUE OCCIDENTALE D‘AVANT LA PÉRIODE AGRICOLE
}

Il y a plus de cent ans, le naturaliste anglais A. Wallace décrivait en ces termes la forêt équatoriale: "Le globe terrestre est ceint à la hauteur de l'équateur d'une zone ininterrompue de la forêt toujours verte dont la largeur atteint 500 1000 lieues (...) C'est un univers où l'homme se sent étranger, écrasé par la présence des forces de la nature qui, des éléments simples de l'atmosphère, ont créé cet océan de verdure ombrageant et étouffant la terre" (Wallace, 1956, p. 43). Hélas, les forces de la nature se sont montrées trop faibles face à l'homme et ses activités destructrices. Aujourd'hui, ce n'est pas celui-ci qui est écrasé par la forêt, comme l'écrivait Wallace, mais c'est la forêt qui recule devant les activités économiques de l'homme. Comme le prouvent des botanistes et des géographes contemporains, même à l'époque où Wallace écrivait ces mots, dans la seconde moitié du XIX ${ }^{e}$ s., la forêt équatoriale avait déjà subi des transformations dues à l'agriculture. Aujourd'hui, cette formation végétale couvre seulement 30-40\% de son ancienne étendue. Les processus de dégradation de la forêt dense humide ont été particulièrement intenses en Afrique Occidentale.

Il serait intéressant de délimiter la superficie qui était couverte de la forêt dense humide à l'époque où l'homme n'était qu'aux débuts de ses activités agricoles - il s'agit de l'étendue de la forêt d'avant l'intervention humaine. La comparaison de l'ancienne superficie couverte de cette formation végétale avec la superficie actuelle permettrait de nous faire une idée sur la dévastation due aux activités économiques de l'homme. Ainsi, nous pouvons nous demander si la responsabilité de la régression de la forêt dense humide aux temps historiques revient à l'homme et à lui seul. La réponse semble être affirmative car de l'avis de nombreux savants, après la période instable du Pléistocène et les phases initiales de l'Holocène, le climat de l'Afrique s'est stabilisé il y a 2,5-3,0 mille ans.

Il semble que la meilleure méthode de définir les conditions climatiques indispensables à une formation végétale consisterait à déterminer la durée de la période de croissance des végétaux. On y recourt de plus en plus souvent ce qui est signifié dans de nombreux documents de la FAO. La période de croissance c'est cette partie de l'année où s'opère le développement physiologique des 


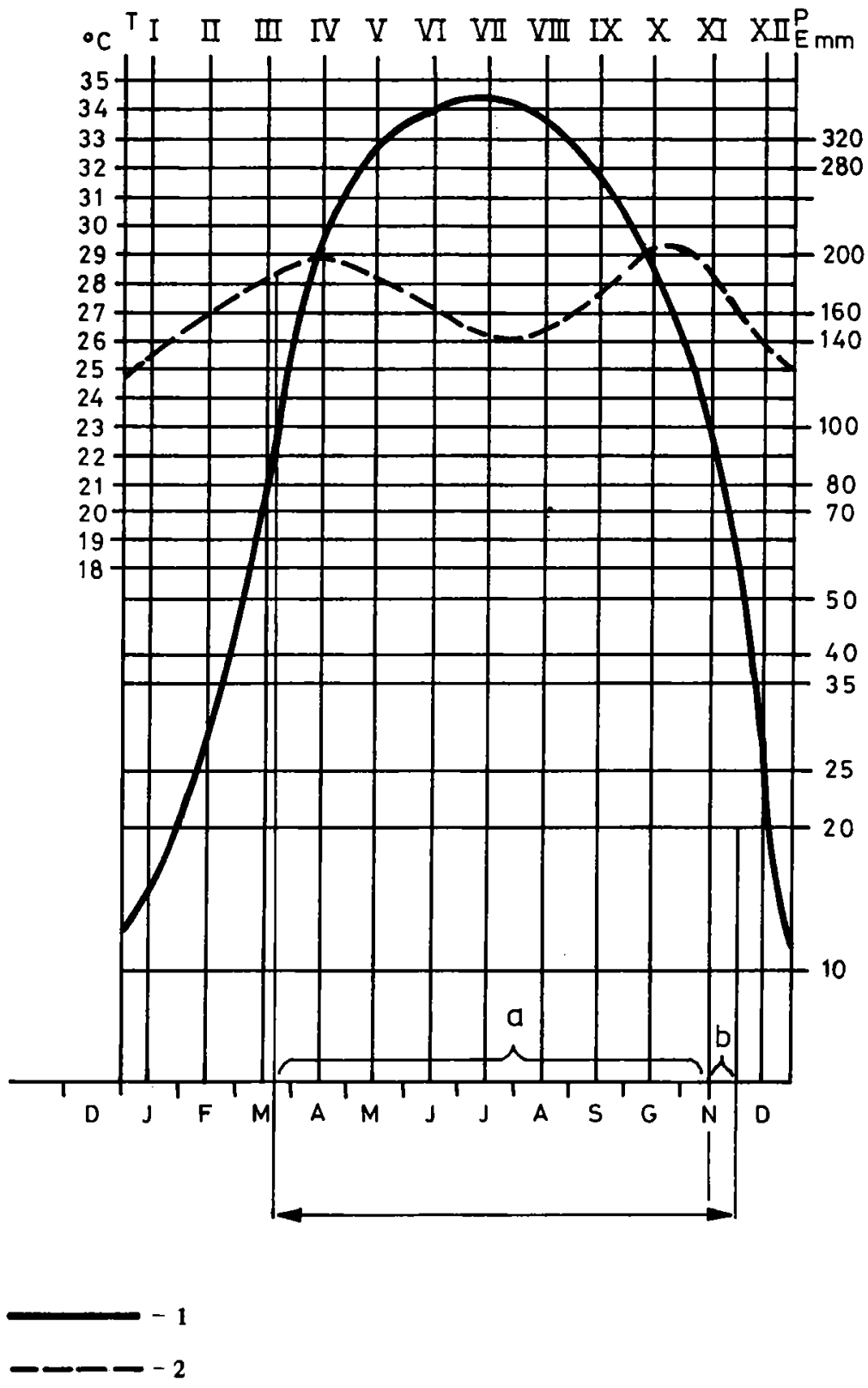

Fig. 1. Méthode d'évaluation de la durée de la période de croissance des végétaux

1 - précipitations, 2 - ETP; a - péiode où $P>0.5$ ETP, b - 15 jours supplémentaires où les plantes absorbent de l'eau du sol 


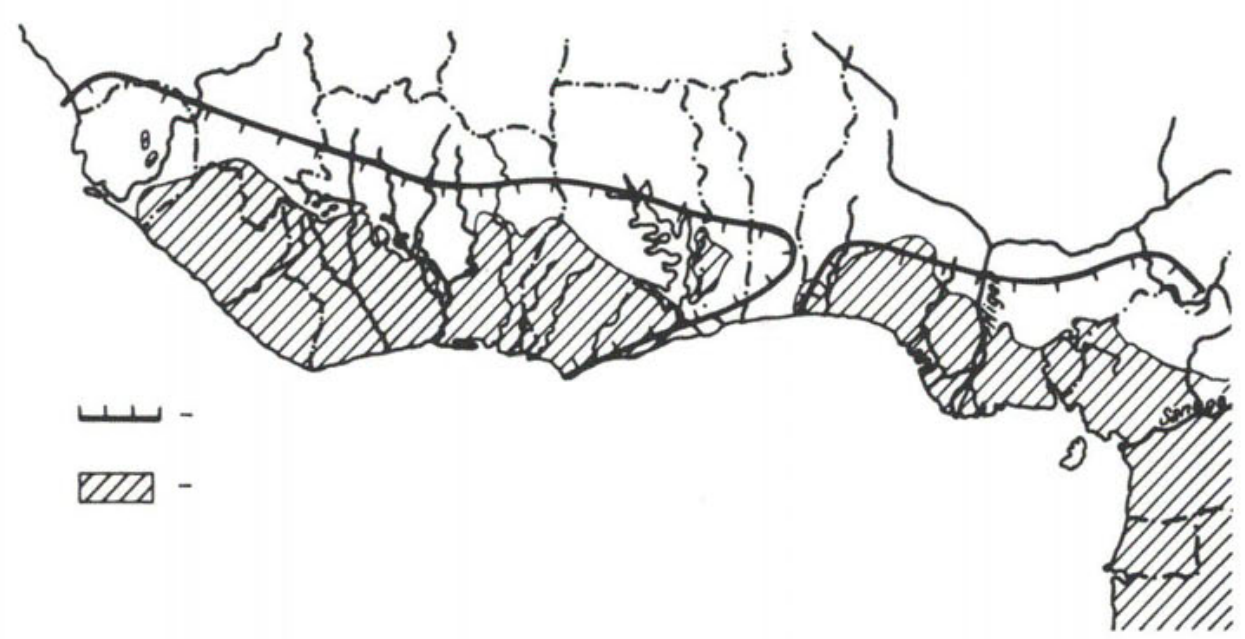

Fig. 2. Aires d'extension de la forêt dense humide en Afrique Occidentale.

1 - limite de la forêt d'avant la période agricole, 2 - aire contemporaine, potentielle (F.White 1986).

végétaux. Dans la zone équatoriale, cette période commence au moment où la plante est capable d'absorber du sol une quantité d'eau indispensable à son développement et finit lorsque la quantité d'eau accessible devient insuffisante.

La durée de la période où la plante dispose d'une quantité d'eau suffisante peut être évaluée grâce à une comparaison des diagrammes de l'évapotranspiration potentielle annuelle avec ceux des précipitations. L'évapotranspiration potentielle peut être établie à partir des abaques hydrothermiques proposées par G.Euverte (1959) d'après le principe de W.Köppen selon lequel il y a une interdépendance entre les besoins en eau des plantes cultivées et la température de l'air. Lesdits besoins (appelés plus tard évapotranspiration - ETP) doublent avec l'accroissement de la température mensuelle de $6^{\circ} \mathrm{C}$ - pour la tempérture mensuelle de $20^{\circ} \mathrm{C}$, les besoins en eau sont estimés à $70 \mathrm{~mm}$ ETP. Ainsi, en traçant sur le diagramme la courbe des températures mensuelles d'une station donnée, nous obtenons en même temps la courbe de l'ETP. Et en traçant sur le même diagramme la courbe de la pluviosité mensuelle, nous pouvons comparer les données obtenues. La période de croissance des végétaux commence au moment où les précipitations dépassent la valeur de $50 \%$ de l'ETP (P > 0,5 ETP) et finit lorsque les précipitations n'atteignent pas $50 \%$ de la valeur de l'ETP (P $<0,5$ ETP). A la durée de cette période A.Kawalec ajoute 15 jours supplémentaires où les plantes absorbent de l'eau du sol et continuent de fonctionner normalement en dépit des précipitations moins importantes.

De nombreux auteurs et ouvrages (dont A.Kawalec 1980; Zaïre...1987) ont attiré l'attention sur une relation entre la durée de la période de croissance des végétaux et l'aire d'une formation végétale donnée. A leur avis, la forêt dense humide peut vivre dans la zone où la PCV n'est pas moindre de 300 jours tandis que là où celle-ci se limite à 270 jours, il y a, par endroits, la forêt semi- 
caducifoliée. Ainsi, l'isoligne de 270 jours de la PCV correspondrait à l'aire potentielle de la formation végétale qui nous intéresse, dans les conditions climatiques actuelles. L'étude de ce problème poursuivie en République Centrafricaine où l'environnement naturel dont la végétation a moins souffert du fait de l'intervention humaine par rapport à l'Afrique Occidentale, a confirmé cette dépendance.

Si nous essayons de délimiter une aire théorique de la forêt dense humide d'avant la période agricole, nous sommes obligés de recourir à une modification. Les spécialistes sont tous d'accord que la dégradation de la végétation forestière entraîne les variations du climat. Les recherches menées par nombre d'entre eux (dont J.Harroy, 1949; E.Salati, T.E.Lovejoy, B.P.Vase 1983; L.A.Bruijnzeel, 1990) ont démontré que de la dégradation de la végétation forestière résulte une baisse importante des précipitations de convexion. Dans les terrains déboisés, la baisse des précipitations peut atteindre de $12,5 \%$ jusqu'à $50 \%$ Ainsi, si nous procédons à la délimitation théorique de la forêt dense humide en évaluant la durée de la PCV, nous devons augmenter les valeurs des précipitations pour les stations situées dans les terrains déboisés soit dans une savane guinéenne d'aujourd'hui.

La valeur de l'augmentation proposée de la pluviosité pourrait faire l'objet des discussions, car la baisse de la hauteur des précipitations restait en rapport avec le caractère d'un site dégradé et était différente dans divers endroits. La valeur de la baisse des pluies ne pouvait être déterminée qu'à l'aide des observations météorologiques de longue durée. Ainsi, faute de données exactes, qu'il nous soit permis d'augmenter les valeurs des précipitations dans des stations de la savane guinéenne de $15 \%$ seulement ce qui est proche de la limite minimum, bien que, probablement, ces valeurs restent en-dessous de la réalité. Les données ainsi modifiées nous permettent d'évaluer, sur l'abaque selon Euverte, la durée de la PCV pour chaque station météorologique. L'isoligne 270 de la PCV délimiterait donc l'aire théorique de la forêt dense humide d'il y a $2500-3$ mille ans.

La méthode présentée ci-dessus de délimiter l'aire de la végétation historique est certes assez controversée, ne serait-ce que du fait de son côté subjectif, ainsi l'aire en question pourrait être considérée uniquement comme approximative. Néanmoins, il nous semble qu'en dépit de toutes ses imperfections, cette méthode pourrait servir de point de départ aux études plus détaillées.

\section{BIBLIOGRAPHIE}

B ruijnzeel L.A., 1990, Hydrology of Moist Tropical Forests and Effects of Conversion: a State of Knowledge Review, Amsterdam.

E u v r te G., 1959, Les Climats en agriculture, PUF, Paris.

H a r ro y J.P., 1949, Afrique - terre qui meurt, Bruxelles.

Kawalec A., 1980. Les Zones agroécologiques de la République Centrafricaine. Méthodologie - limites des zones, FAO, Bangui.

S a lati, E., Lovej oy T.E., Vos e P.B., 1983, "Precipitations and Water Recycling in Tropical Rain Forests with Special Reference to the Amazon Basin, Environmentalist, 1.

Wh i te F., 1986, La Végétation de l'Afrique, UNESCO, Paris.

Zaïre, projet de développement agricole au Kivu, 1987, FAO, Rome.

W all a ce A.R., 1956, Tropiceskaïa priroda, Moskva. 